for the profession to work together in the future. Facilitating and coordinating such working, including relevant expert input, is a key aim of the new College (https:// cgdent.uk). As an independent, standard setting, UK-wide organisation for all members of the dental team, the College will be best placed to provide the leadership Dr Mannan correctly identifies as missing.

To enable the College to become fully established at the earliest possible opportunity and provide the collegiate leadership which the dental profession requires, all dental healthcare professionals, who have not already done so, are encouraged to become a Foundation Member of the College - a quick, simple, inexpensive online process (https://cgdent. uk/join/). Dentistry must no longer be the only major healthcare profession in the UK without a College, let alone a Royal College. N. Wilson, Chair, Board of Trustees, College of General Dentistry, London, UK https://doi.org/10.1038/s41415-020-2069-6

\section{Temperature and mouth opening}

Sir, during the pandemic, facial swellings which do not require an urgent referral to a local maxillofacial unit may initially be managed in practice or by an urgent dental care centre through use of the AAA approach (advice, analgesia and/or antimicrobials) or source control through pulp extirpation, extraction or incision and drainage. The Scottish Dental Clinical Effectiveness Programme (SDCEP) recommends that patients treated for these conditions are reviewed within two to seven days. ${ }^{1}$

In the secondary care environment, haematological and biochemical investigations can be undertaken alongside clinical examination to provide reassurance that the patient's condition is improving following treatment. These tests are not available to the primary care dentist; however, there are two objective, quantifiable measurements which may be used, namely temperature and mouth opening.

Patient-reported symptoms of fever correlate poorly with measured temperature. ${ }^{2}$ Therefore, SDCEP recommends temperature measurements for all patients with suspected bacterial infections. ${ }^{1}$ Some dental practices may have recently invested in temperature screening products, although the Medicines and Healthcare products Regulatory Agency (MHRA) advises caution when interpreting readings from devices that measure skin temperature. ${ }^{3}$ There may be value in utilising these temperature screening products, or preferably thermometers which measure core temperature for assessment of patients with facial swellings even after the pandemic restrictions are eased.

Restriction of mouth opening may result from infection in the masticatory soft tissue spaces (trismus), or as a product of pain (guarding). Severe trismus in the context of infection demands an emergency referral. Measurement of the number of fingers that can be inserted into the mouth is imprecise and lacks reproducibility, and therefore use of a sterilisable or disposable ruler is recommended to evaluate the inter-incisal distance in millimetres. ${ }^{4}$

Thermometers and stainless steel rulers are an inexpensive but valuable addition to the general practitioner's armamentarium and may be particularly useful during the current period of increased emergency care provision.

\section{D. Simpson, Livingston, UK}

\section{References}

1. Scottish Dental Clinical Effectiveness Programme. Drug prescribing for dentistry. 3rd edition. Dundee, UK, 2016.

2. Kass-Hout T A, Buckeridge D, Brownstein J et al. Self-reported fever and measured temperature in emergency department records used for syndromic surveillance. J Am Med Inform Assoc 2012; 19: 775-776

3. Medicines and Healthcare products Regulatory Agency. Don't rely on temperature screening products for detection of coronavirus (COVID-19), says MHRA. 2020. Available at: https://www.gov.uk/government/ news/dont-rely-on-temperature-screening-productsfor-detection-of-coronavirus-covid-19-says-mhra (accessed August 2020).

4. Rapidis A D, Dijkstra P U, Roodenburg J L N et al. Trismus in patients with head and neck cancer: Etiopathogenesis, diagnosis and management. Clin Otolaryngol 2015; 40: 516-526.

https://doi.org/10.1038/s41415-020-2070-0

\section{Dental team immunisation}

Sir, this year, in order to reduce the risk of the dual threat of seasonal influenza and coronavirus, the UK government has expanded the eligibility criteria for flu vaccinations to include those over 50 years of age, shielding households and all school year groups up to year 7 .
This represents approximately 30 million people. ${ }^{1}$ The operational challenges provide an opportunity for innovative models of delivery. Underpinned by collaborative discussions between various organisations we propose potential models to support the delivery of this enhanced programme:

- Foundation dentists to provide additional immunisation capacity

- Dental team members to deliver vaccinations with Primary Care Network (PCN) teams or School Aged Flu Provider workforce in school, primary care and/or community settings

- Use of dental surgery premises by immunisation teams to complement existing community settings that are known and accessible to the public

- Flu vaccination in a dental setting. This could reduce the number of contacts between the public and clinical settings and could be done for specific cohorts and/or opportunistically.

Another avenue is the potential for dental workforce vaccinators to support the catch up of adolescent immunisation programmes such as HPV and meningitis, delayed due to pandemic school closures. Once a safe and effective vaccine for COVID-19 becomes available, it is expected that delivering a large-scale immunisation programme will raise significant logistical issues. If the above models have evaluated well, dental care providers would be well placed to support the delivery of such programmes.

Any of the above models would need to take into consideration certain factors such as training, competency sign-off, indemnity, funding and regulatory approval. There is now a short window of opportunity before the beginning of the flu season to explore these possibilities but the lessons learned from the pandemic response might provide an opportunity to strengthen the dialogue between medicine and dentistry.

S. Serban, S. Eapen-Simon, S. Grant, Leeds, UK

\section{Reference}

1. UK Government Press Office. Most comprehensive flu programme in UK history will be rolled out this winter 2020. Available at: https://www.gov.uk/government/ news/most-comprehensive-flu-programme-in-ukhistory-will-be-rolled-out-this-winter (accessed July 2020).

https://doi.org/10.1038/s41415-020-2071-z 